\title{
Case Report of Schwannomas: Benign Tumour of the Peripheral Nerve Sheath
}

\author{
Authors: \\ *Ansilata Marlyn Anesly, ${ }^{1}$ Faisal Ameer, ${ }^{1}$ Hillol Kanti Pal ${ }^{2}$ \\ 1. Plastic Surgery, Thumbay Hospital, Ajman, United Arab Emirates \\ 2. Neurosurgery, Thumbay Hospital, Ajman, United Arab Emirates \\ *Correspondence to ansilatamerlyn025@gmail.com \\ Disclosure: $\quad$ The authors have declared no conflicts of interest. \\ Received: \\ 16.12 .19 \\ Accepted: \\ 21.02.20 \\ Keywords: \\ Benign, peripheral nerve sheath, schwannoma, solitary, sural nerve, tibial nerve. \\ Citation: \\ EMJ Neurol. 2020;8[1]:103-107.
}

\section{Abstract}

Schwannomas, also known as neurilemmomas, are benign peripheral nerve sheath tumours arising from the Schwann cells surrounding the nerve. Incidence of peripheral nerve sheath benign tumours occurring in the lower extremities is rare (1-10\%). The authors present two cases with solitary schwannomas from the peripheral nerves. In one case, the schwannoma arose from the sural nerve and in the other, from the tibial nerve. They were successfully surgically removed with the aid of a surgical microscope, with no intraoperative or postoperative complications.

\section{INTRODUCTION}

\section{CASE PRESENTATION 1}

Schwannomas are benign, painless, firm nodules, 1-2 cm in diameter, that develop from the Schwann cells of peripheral nerve sheaths. They are tethered to a nerve and are therefore only laterally mobile. They are asymptomatic and nontender, and are not associated with any nodal involvement or malignant potential. The diagnosis is usually made histologically after excision of the tumour. $^{1}$ Malignant transformation of schwannomas is extremely rare..$^{2-5}$ Histologically, schwannomas are differentiated by the presence of a highly cellular Antoni A component that palisades Verocay bodies and myxoid hypocellular Antoni B components. These cases provide an insight into diagnosis and management of solitary schwannomas originating from peripheral nerves in adult males.
A 39-year-old male presented with tender, palpable swelling and pain on the lateral aspect of the right mid-calf, shooting down the lateral aspect of the right leg to the ankle for 2 months. Physical examination revealed a tender, solid subcutaneous mass at mid-calf level measuring $20.0 \times 20.0 \times 15.0 \mathrm{~mm}$, with no focal neurological deficits. The patient's past medical history was not significant. MRI of the right leg showed a welldefined, ovoid soft tissue mass lesion seen in the posterior aspect of the right leg. The lesion lay directly posterior to the myotendinous junction of the gastrocnemius muscle, causing overlying skin bulge. It measured approximately $14.3 \times 13.7 \times 11.1$ $\mathrm{mm}$ at its maximal craniocaudal length (Figure 1). A benign nerve sheath tumour, such as schwannoma, was strongly suspected. 

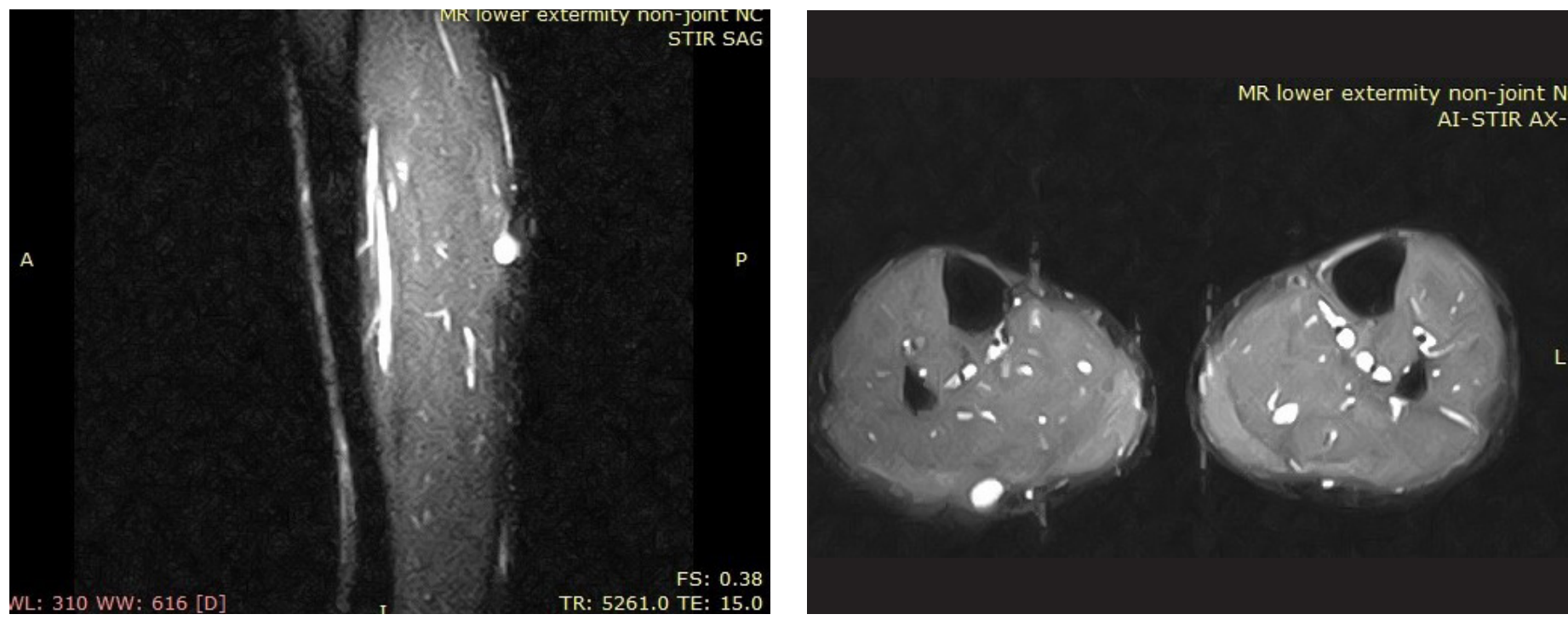

Figure 1: MRI revealed a well-defined, oval mass in the posterior aspect of the right leg.
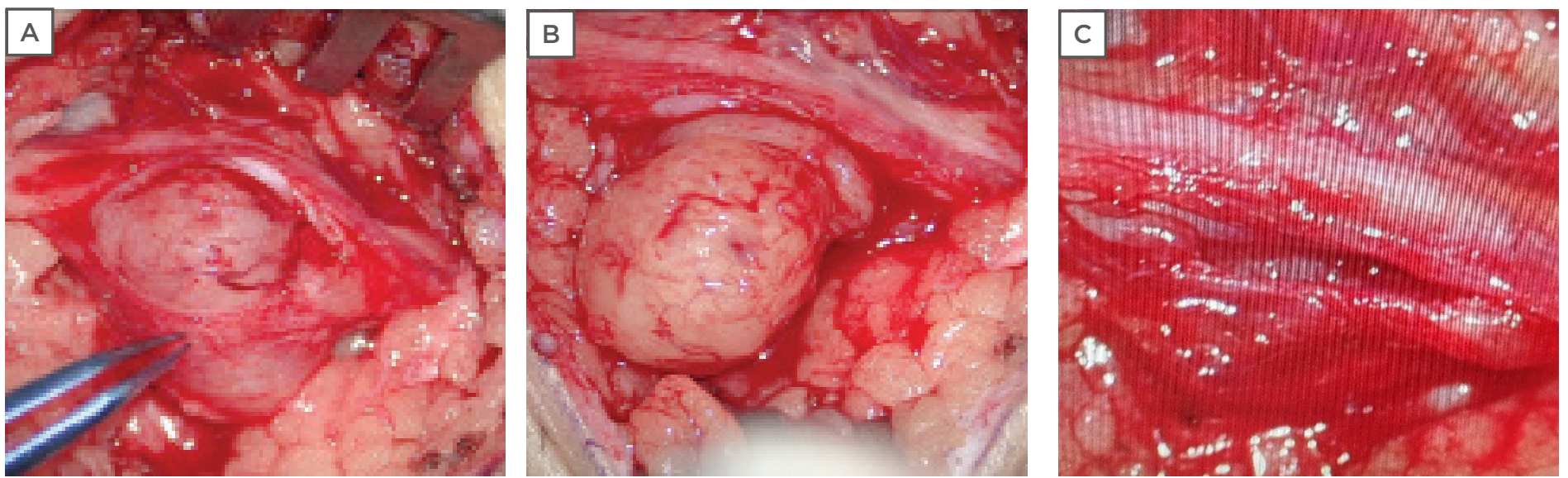

Figure 2: A) Isolation of the tumour with incision of the capsule; B) gradual enucleation of the tumour with nerve fibres intact; C) postexcision of schwannoma with intact sural nerve.
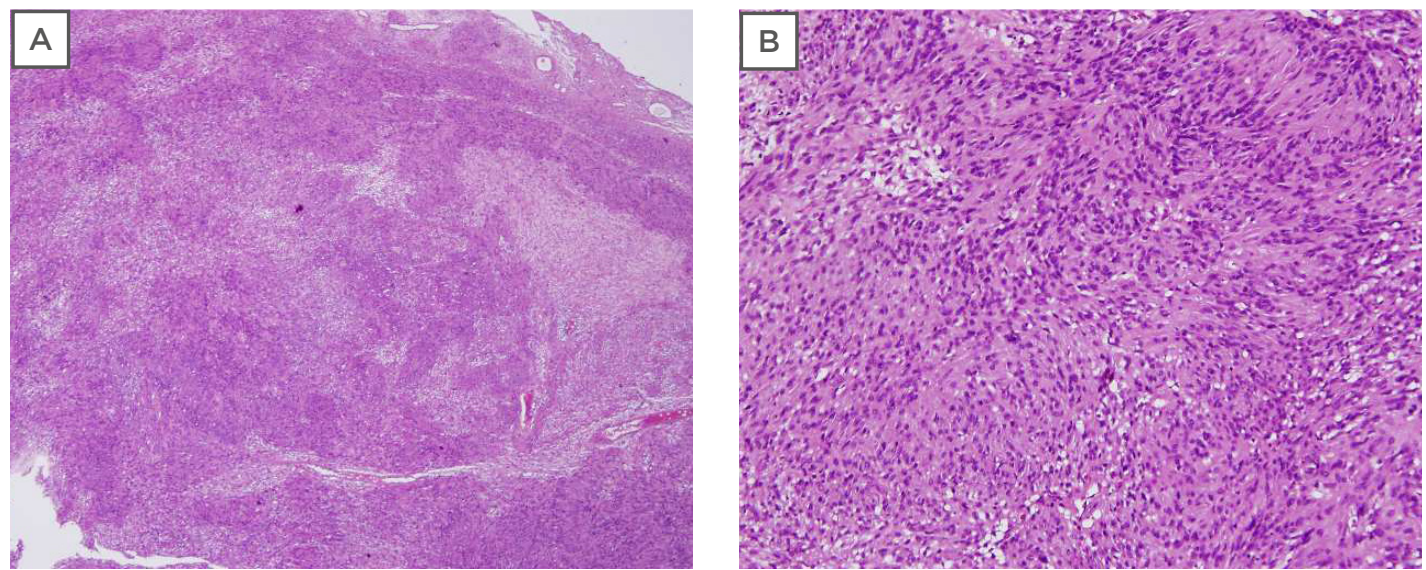

Figure 3: Histopathologic examination shows hypocellular Antoni (A) and hypercellular Antoni (B) Verocay bodies, confirming the diagnosis of benign schwannoma. 


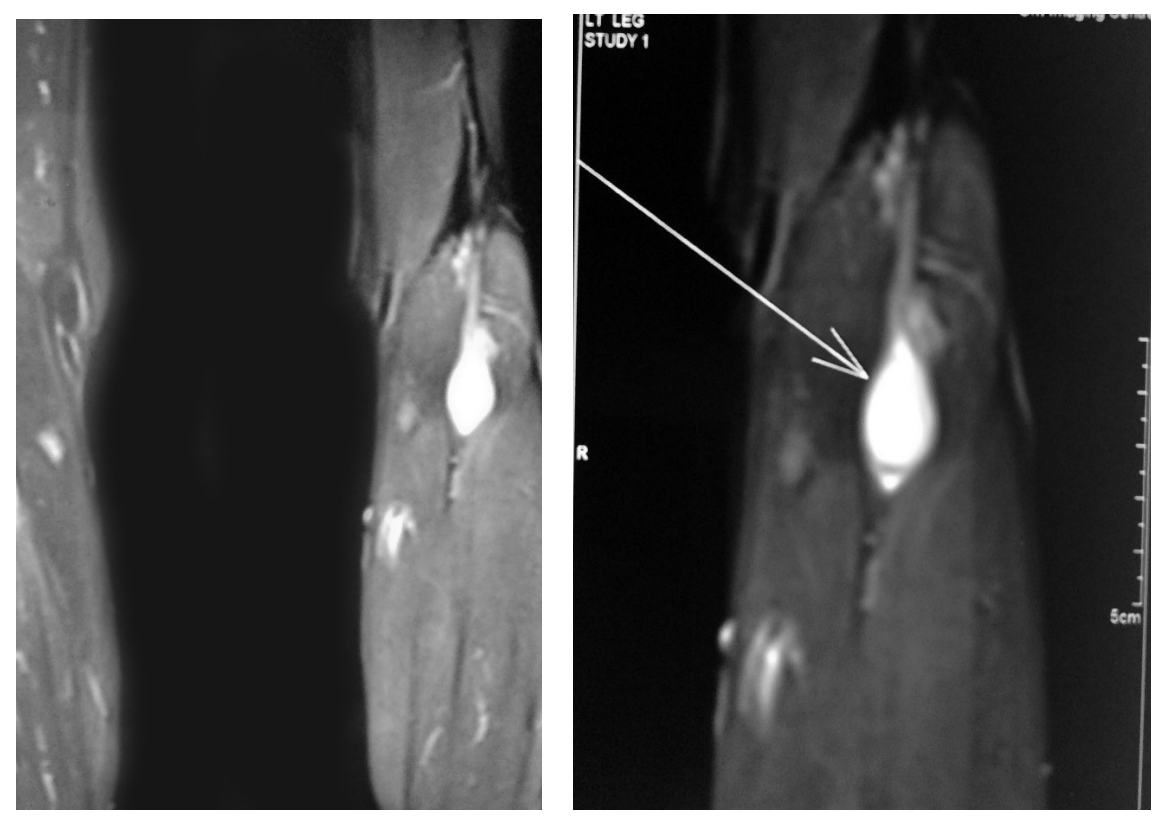

Figure 4: Well-defined, smooth, oval mass (depicted by arrow) with involvement of the posterior tibial nerve.
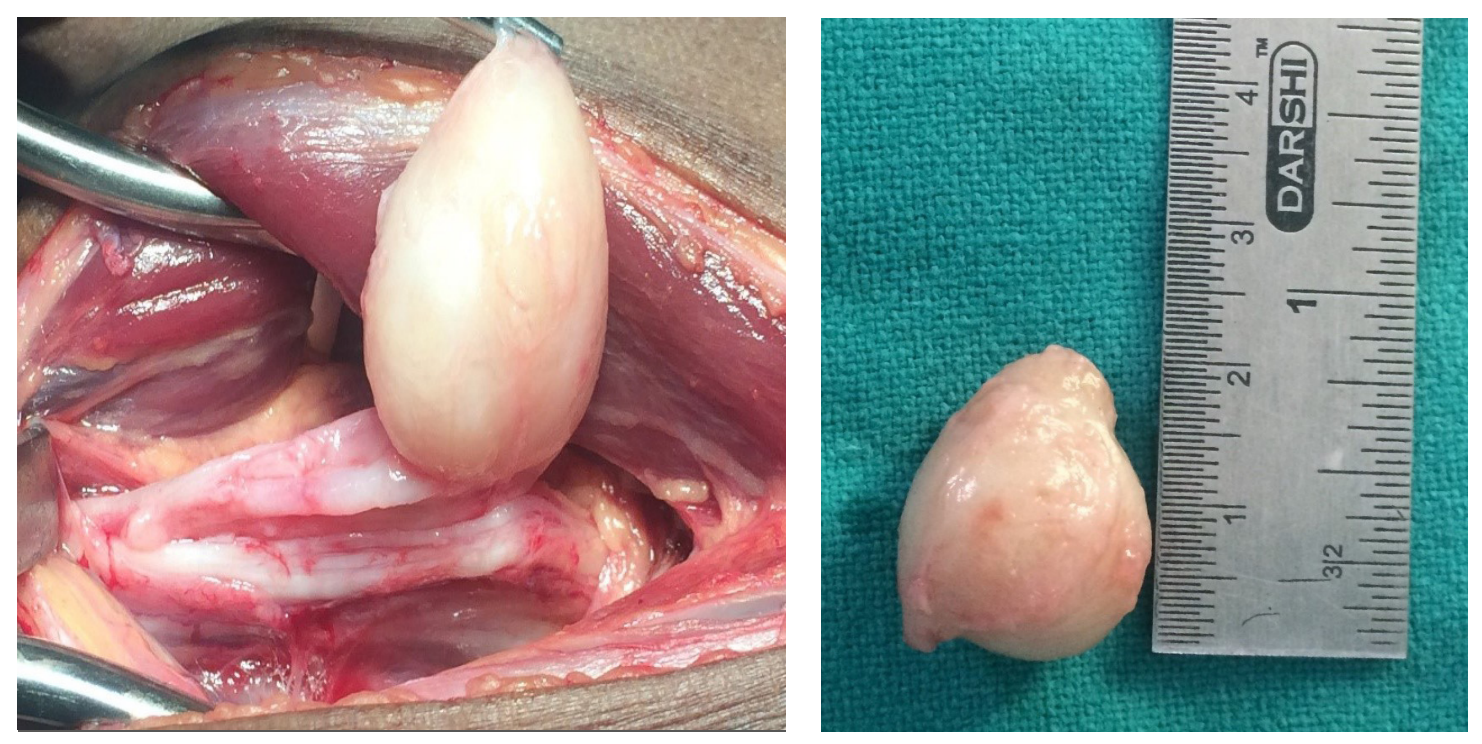

Figure 5: A) Complete enucleation of the schwannoma with nerve fibres intact; B) enucleated schwannoma measuring $22 \mathrm{~mm}$.

The surgery was performed under local anaesthesia. Lidocaine $2 \%$ with adrenaline was injected subcutaneously at the site of the tumour. A 'lazy S' incision was made at the site to allow for exposure of the tumour to the level of deep fascia. The Capsule of the tumour was identified and gradually incised to dissect away the encapsulated tumour using sharp dissection. One fascicle of nerve was identified as entering the tumour, and was carefully excised. The tumour was completely removed and haemostasis was achieved. The wound was closed in layers with Monocryl ${ }^{\circ}$ 5-O sutures. Grossly, the tumour was firm, smooth-surfaced, and appeared pale yellow in colour (Figure 2A, B, C). Histopathology of the excisional biopsy confirmed benign schwannoma (Figure 3). The postoperative phase was uneventful. 


\section{CASE PRESENTATION 2}

A similar case of a 40-year-old male presented with pain and claudication associated with a tender swelling on the posterior aspect of the upper calf of the left leg. Examination revealed a firm mass, mobile laterally, on the posterior aspect of the left leg, measuring approximately $2.5 \mathrm{~cm}$ in diameter.

MRI revealed a well-encapsulated oval mass in the posterior compartment of the leg: located in the infrapopliteal region. It showed marked T2-weighted hyperintense signal with smooth perilesional planes. Contrast-enhanced MRI showed homogenous marked enhancement in the lesion (Figure 4).

On exploration under regional anaesthesia and tourniquet control, the tumour was identified deep to gastrocnemius soleus, closely associated with fibres of the tibial nerve. The perineural sheath was carefully dissected under magnification, with preservation of all nerve fibres and complete tumour excision (Figure 5A, B). Histopathologic examination revealed benign schwannoma. Postoperatively, the patient had no complaints of sensory or motor weakness.

\section{DISCUSSION}

The differential diagnoses of such cases include neurofibroma and benign vascular tumours such as angioleiomyoma. Cystic hygroma, followed by lipoma, haemangioma, ganglion, and myxoma, are also commonly seen. ${ }^{6}$ The sural nerve is a sensory nerve that passes down the posterolateral side of the leg and on to the dorsal aspect of the lateral side of the foot. The sural nerve runs with the small saphenous vein on the posterior leg, just lateral to the Achilles tendon. Its terminal branches consist of the lateral dorsal cutaneous nerve and the lateral calcaneal branches, whereas the tibial nerve branch of the sciatic nerve follows a course down the leg posterior to the tibia. It provides sensory innervation to the posterolateral side of the leg, the lateral side of the foot, and sole of the foot, and motor innervation to the posterior compartment of the leg. A solitary schwannoma arising from the tibial nerve is more common than from the sural nerve, which is reported to be a rare occurrence. ${ }^{7-12}$ Despite its rare occurrence, schwannoma should always be included in the differential diagnosis.

Schwannomas or neurilemmomas are wellencapsulated, lobulated lesions without metastatic potential, particularly if they are located in the extremities. The lesions are mostly solitary, slow-growing, painless tumours and malignant transformation is a rare event. Schwannomas do not transverse through the nerve but remain in the sheath, unlike neurofibromas. Schwannomas can cause distal symptoms if found in the proximal aspect of the lower limbs. ${ }^{13,14}$ Permanent nerve damage and soft tissue and bone deformity can be prevented by early diagnosis. MRI and sonography can help diagnose these lesions early. ${ }^{15,16}$ The aim of treatment is total excision of the lesion without causing nerve damage that may lead to sensorimotor deficit. ${ }^{17,18}$ In these cases, good magnification and good microsurgical technique under tourniquet control was extremely important to achieve this goal.

\section{CONCLUSION}

This report focusses on the early diagnosis of schwannoma by performing an MRI of the lower extremities. The cases were managed by complete excision of the benign tumour, preserving the peripheral nerve fibres with the aid of a surgical microscope, which provided better magnification of the operating field and meticulous dissection of the mass.

\section{References}

1. Sturzenegger $M$ et al. [Microsurgical and histological observations in schwannoma of peripheral nerves]. Handchir Mikrochir Plast Chir. 1992;24(6):304-9. (In German).

2. Robey SS et al. Malignant epithelioid peripheral nerve sheath tumour arising in a benign schwannoma. 1987;28(6):441-6

3. Rober PE et al. Malignant peripheral nerve sheath tumor (malignant schwannoma) of urinary bladder in von Recklinghausen neurofibromatosis. Urology. 1991;38(5):473-6

4. Mikami $Y$ et al. Malignant peripheral nerve sheath tumor arising in benign ancient schwannoma: a case report 
with an immunohistochemical study. Pathol Int. 2000;50(2):156-61.

5. Robson DK, Ironside JW. Malignant peripheral nerve sheath tumour arising in a schwannoma. Histopathology. 1990;16(3):295-7.

6. Rabari D et al. Schwannomas - the problem in delay diagnosis. Int $J$ Orthop Sci. 2017;3(1g):459-62.

7. Yamamoto $\mathrm{K}$ et al. Solitary schwannoma of the sural nerve: an unusual clinical presentation. Exp Ther Med. 2014;7(1):90-2.

8. Tourne $Y$ et al. [A case of benign schwannoma of the posterior tibial nerve. Treatment by simple excision without nerve graft]. J Chir (Paris). 1989;126(3):204-5. (In French).

9. Tladi MJ et al. Schwannoma and neurofibroma of the posterior tibial nerve presenting as tarsal tunnel syndrome: review of the literature with two case reports. Foot (Edinb).

$$
2017 ; 32: 22-6 \text {. }
$$

10. Herrero San Martín A, AlcaláGaliano A. Schwannoma of the posterior tibial nerve in a patient with schwannomatosis and a novel mutation of the LZTR1 gene. Neurologia. 2019;SO2134853(19)30114-8. [Epub ahead of print].

11. Gharwade CR et al. Sural nerve schwannoma: a case report. Int Surg J. $2015 ; 2(4): 746-8$

12. Buchanan V, Rawat M. Schwannoma of the posterior tibial nerve. J Orthop Sports Phys Ther. 2020;50(2):111.

13. Rajasekaran RB, Shanmuganathan R. Schwannoma of the posterior tibial nerve presenting as tarsal tunnel syndrome: a case report with emphasis on the role of microscope during surgery. Case Rep Orthop. 2018:4704362.

14. Hallahan $\mathrm{K}$ et al. Tarsal tunnel syndrome secondary to schwannoma of the posterior tibial nerve. J Foot Ankle Surg. 2014;53(1):79-82.

15. Lee JA, Boles CA. Peripheral schwannoma lacking enhancement on MRI. AJR Am J Roentgenol. 2004;182(2):534-5.

16. Lebreton $\mathrm{E}$ et al. [Schwannoma of the peripheral nerves. Their localization and treatment]. Ann Chir Plast Esthet. 1985;30(4):309-15. (In French).

17. Hara M et al. [Surgical Technique for Peripheral Nerve Schwannoma Resection]. No Shinkei Geka. 2019;47(12):1223-9. (In Japanese).

18. Bendon $\mathrm{CL}$ et al. Comparison of outcomes of peripheral nerve schwannoma excision in neurofibromatosis Type 2 patients and non-neurofibromatosis type 2 patients: a case control study. $J$ Plast Reconstr Aesthet Surg. 2015;68(9):1199-203. 